\title{
An imaging algorithm of objects embedded in a lossy-dispersive medium for subsurface radar-data processing
}

\author{
$\operatorname{AUTHOR}(\mathrm{S})$ : \\ Sato, T; Wakayama, T; Takemura, K
}

\section{CITATION:}

Sato, T ...[et al]. An imaging algorithm of objects embedded in a lossy-dispersive medium for subsurface radar-data processing. IEEE TRANSACTIONS ON GEOSCIENCE AND REMOTE SENSING 2000, 38(1): 296-303

\section{ISSUE DATE:}

2000-01

URL:

http://hdl.handle.net/2433/50299

\section{RIGHT:}

(c)2000 IEEE. Personal use of this material is permitted. However, permission to reprint/republish this material for advertising or promotional purposes or for creating new collective works for resale or redistribution to servers or lists, or to reuse any copyrighted component of this work in other works must be obtained from the IEEE. 


\title{
An Imaging Algorithm of Objects Embedded in a Lossy-Dispersive Medium for Subsurface Radar-Data Processing
}

\author{
Toru Sato, Member, IEEE, Toshio Wakayama, and Kazuhisa Takemura
}

\begin{abstract}
A robust and high-resolution two-dimensional (2-D) imaging algorithm is proposed for retrieving the shape of conductive objects embedded in a uniform lossy and dispersive medium. The target is modeled in terms of the location of points that represents the outer contour of the object, together with the parameters of the medium. A nonlinear least-squares fitting is applied to the estimated scattered waveform to adjust the model parameters. The estimated waveform is computed using the extended ray-tracing method, which incorporates the edge-diffraction waves. The performance of the algorithm is examined with numerical simulations and test-site experiments. The simulation with clutters also revealed the robustness of the algorithm even under fairly strong clutter environments.
\end{abstract}

Index Terms-Clutter rejection, radar imaging, subsurface radar data processing.

\section{INTRODUCTION}

$\mathbf{I}^{\prime}$ n subsurface radar applications, it is often found that the requirements for the resolution and the penetration depth contradict each other. For example, preexcavation surveys of archaeological sites may require the detection of buried objects of the order of $10 \mathrm{~cm}$ at a depth of $1 \mathrm{~m}$. In such a case, the compromise will be the use of approximately $500 \mathrm{MHz}$, for which the target is on the order of a radar wavelength. We refer to the target in such a situation as a "small object." It is thus hard to identify the shape of the object with conventional signal-processing techniques.

Shape estimation is among the most important issues in radar signal processing. A number of techniques have been developed to retrieve the shape information from the received signal. When the target can be observed from a wide angular range, the synthetic aperture radar (SAR) or the inverse SAR techniques [1] provide stable two-dimensional (2-D) images. The singularity expansion method (SEM)[2] relates the target shape to the signal waveform in a monostatic situation.

These techniques assume electrically large targets, and thus have limited applicability to the subsurface radar signal pro-

Manuscript received September 8, 1998; revised March 23, 1999.

T. Sato is with the Department of Communications and Computer Engineering, Kyoto University, Kyoto 606-8501, Japan (e-mail: tsato@kuee.kyoto-u.ac.jp).

T. Wakayama was with the Department of Electronics and Communication Engineering, Kyoto University, Kyoto 606-8501, Japan. He is now with the Mitsubishi Electric Corporation, Tsukaguchi, Amagasaki, Japan.

K. Takemura was with with the Department of Electronics and Communication Engineering, Kyoto University, Kyoto 606-8501, Japan. He is now with Kansai Electric Power Co. Ltd, Osaka, Japan.

Publisher Item Identifier S 0196-2892(00)00012-7. cessing. In recent years, special techniques have been developed to solve the inverse problems of subsurface-radar applications. One group of studies aims to reconstruct the 2-D or three-dimensional (3-D) profile of the inhomogeneous ground. The iterative Born method is limited to a situation of a fairly narrow range of the medium parameter, but provides a stable image with the aid of a constrained optimization [3]. A nonlinear scattering approximation was introduced to relax the restriction of the iterative Born method [4], although it requires a wide angle of illumination, as is the case of crosswell borehole radar, in order to obtain a stable image.

Another group of study is motivated to locate and determine the shape of a more high-contrasted targets such as metallic objects. They include a model-based algorithm based on the diffraction tomography [5] and a quasiexact active-imaging method [6]. These methods do not require assumptions on the target shape but are restricted to the case where the medium is uniform and nondispersive.

Among the features that specialize subsurface radar, the loss and dispersion of the medium, together with the existence of strong clutters, strictly limit the usefulness of these techniques. For an accurate imaging of subsurface objects, it is thus essential to develop an algorithm that can handle these features. While it is hard to include the effect of loss and dispersion directly in inverse scattering problems, various numerical procedures have already been developed for the forward scattering cases.

Our approach has been to model the target with a limited number of parameters and to recursively modify them so that the observed signal waveforms fit the estimated ones computed for the model [7], [8]. In this paper, we extend the algorithm to handle a more realistic case of a conductive, finite-sized target embedded in a 2-D homogeneous lossy and dispersive medium.

\section{Discrete Model-FitTING Algorithm}

Our algorithm, which is called the discrete model fitting (DMF) method [7], solves the inverse problem of imaging the subsurface object by iteratively comparing the estimated received-signal waveforms computed from the model of the target with those of the received signal and by adjusting the parameters that describe the model so that the variance between the waveforms is minimized. Currently, it deals with 2-D imaging based on a 1-D (horizontal) scan of the radar sensor, although both monostatic and multistatic cases can be treated.

In the DMF, the target and the medium are described by a parameter vector $\boldsymbol{X}$. The model parameters consist of the loca- 
tion of the points representing the outer contour of the target and the characteristics of the medium. Let the received-signal waveform at the $i$ th receiver position be $e_{i}(t)$, and let the estimated waveform at the same position computed from the target model be $\hat{e}_{i}(t ; X)$. Our problem is to determine $\boldsymbol{X}$ by minimizing the cost function

$$
F(\boldsymbol{X})=\sum_{i=1}^{N}\left\{e_{i}(t)-\hat{e}_{i}(t ; X)\right\}^{2}
$$

where $N$ is the number of observation points.

The cost function $\hat{e}_{i}(t ; \boldsymbol{X})$ is generally nonlinear in terms of $\boldsymbol{X}$, so we linearize it by expanding it around a proper initial guess $\boldsymbol{X}_{0}$

$$
\hat{e}_{i}\left(t ; X_{0}+\Delta \boldsymbol{X}\right) \simeq \hat{e}_{i}\left(t ; \boldsymbol{X}_{0}\right)+\Delta \boldsymbol{X} \cdot \frac{\partial}{\partial \boldsymbol{X}} \hat{e}_{i}(t ; \boldsymbol{X})
$$

We determine $\Delta \boldsymbol{X}$ by the least-squares fitting and then iterate the process by using $\boldsymbol{X}_{0}+\Delta X$ as a new initial guess. Since this iterative procedure is not necessarily stable, we employ the modified Marquardt method [9], which combines the Gauss-Newton method with the steepest decent method, so that stability is assured while keeping the fast convergence.

At the first step, outstanding targets are estimated as a group of point targets. The position of each target is estimated from the peaks in the received-signal waveform, which gives the distance of the points from each antenna. All possible combinations of these points determined from two received signals at different antenna locations are given as intersections of two circles, and are plotted on a plane.

A consensus analysis [7] distinguishes the true points from spurious ones. In order to select correct positions of targets, we define the degree of concentration of the estimated points. The degree of concentration is calculated by counting the neighboring intersections with Gaussian weight at grid points set in the whole observational region, and is given by

$$
C_{j}=\sum_{i=1}^{N} \exp \left\{-\frac{\left(x_{i}-x_{j}\right)^{2}+\left(y_{i}-y_{j}\right)^{2}}{r_{0}^{2}}\right\}
$$

where

$$
\begin{array}{ll}
\left(x_{i}, y_{i}\right) & \text { position of an intersection of two circles; } \\
N & \text { number of intersections; } \\
\left(x_{j}, y_{j}\right) & \text { position of the grid point; } \\
r_{0} & \text { width of the Gaussian weighting function. }
\end{array}
$$

We empirically set $r_{0}=\lambda / 4$ so that it does not reduce the resolution, where $\lambda$ is the radar wavelength at the center frequency of the transmitted pulse.

Discontinuity in the medium can also be estimated based on the consensus analysis of the received time series at each point during the scan (although this is not examined in the current application, in which we assume a uniform medium). Then the nonlinear least-squares fitting is used to improve the model in an iterative manner by estimating the location of the point targets [10].

For each prominent target found in the first step, the shape-estimation procedure is applied. The shape of the target is expressed in terms of the points that represent its outer boundary. The shape estimation starts from the single point found in the

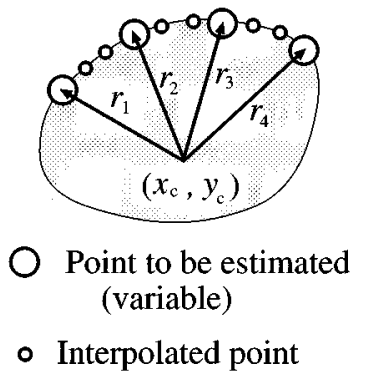

Fig. 1. Arrangements of the variable and interpolated points in modeling the target shape.

first step, and then the algorithm increases the number of points in a stepwise manner. The second point is searched in the two opposite transverse directions, among which, the direction that gives a better fit is chosen. The extension is terminated when the direction of the reflected ray from the extended surface falls away from the receiving-antenna position. Extension of the surface more than this limit usually results in an expansion into the direction from which no echo returns to the direction of antennas.

These points are selected so that the distance between adjacent points is on the order of $\lambda / 10$, and they are connected smoothly. Fig. 1 schematically shows the arrangements of these points. Location of large circles are varied only in the radial direction from its virtual center, which is determined in each iteration, and the location of small circles are computed by Lagrangian interpolation. In this manner, the set of parameters that describes the model shape is minimized to $\left(x_{\mathrm{c}}, y_{\mathrm{c}}\right)$ and $r_{i}$ ( $i=1, \cdots, M)$, where $M$ is the number of variable points (which is four in the case of Fig. 1).

In general, iterative-inversion procedures may have a problem converging to a local minimum rather than the desired solution. In order to prevent this problem, the proposed procedure always starts from a point target and expands the target size in a stepwise manner. At each step of this expansion, the shape of the object is modified only in a radial direction from its virtual center, which is the direction roughly perpendicular to the curve representing the shape of the object. The optimum size that gives the least residual is thus sought by a 1-D grid search taking the object size as the variable. As far as the smooth shape treated here is concerned, we have not experienced the problem of false convergence to a local minimum.

The minimum size that can be estimated by this method is about $\lambda / 2$. Targets smaller than this size are expressed as round objects of roughly $\lambda / 2$ in diameter. The largest size is limited by the number of variable points used to express the object shape. When the number of the points exceeds about 20, the computational time becomes exceedingly large, although the exact upper limit has not been examined. The appropriate target size is thus less than about $2 \lambda$ at the current parameter setting. A larger object may be treated by enlarging the interval between the variable points if its shape is smooth enough.

\section{EXTENDED RAY TRACING}

For each trial in this recursive model fitting process, the entire forward-scattering problem should be solved. Use of the finite 
difference time domain (FDTD) method is too time consuming for this purpose. In order to save the machine time, we compute the estimated scattered wave by using an extended ray-tracing method that includes the effect of diffraction. Unlike conventional ray tracing, in which an individual ray is traced independently, the rays are treated as groups, each of which represents a wave front.

When a ray group reaches a conductive object, individual rays are reflected according to the simple geometrical optics (GO), as is the case for the conventional ray tracing. Since the surface of the object can be determined at any desired point by the Lagrangian interpolation described in the previous section, its local inclination, and thus the direction of each reflected ray, can be readily determined.

The magnitude of the reflected rays is determined using the physical optics (PO) [11]. The PO field at the center frequency of the reflected pulse is computed at a sufficiently distant point from the object, which is defined here as $10 \lambda$ away, and the energy associated with a ray is determined according to the distance between adjacent rays at this point. By this "calibration" procedure, the magnitude of the reflected rays is accurately computed within about $5 \%$ of that computed by the FDTD method even for a small object of the size of the order of $\lambda$.

The diffraction rays from an edge are generated and appended to the outer limits of a ray group each time the ray group that represents a wave front passes the edge. The magnitude of the diffracted rays is also computed by using PO so that they connect smoothly to the reflected wave at the reflection boundary. Although the accuracy of the PO deteriorates for the rays far from the reflection boundary, it does not largely affect the entire estimation procedure, since the magnitude of such rays is small.

Fig. 2 shows an example of the rays thus generated. In the ray tracing, only the location of the wavefront and the energy associated with it is computed at each time step. In order to generate the estimated signal waveform at the location of the receiving antennas, the waveform for the reflected waves is computed once using the FDTD method and convolved with the impulses that represent individual rays corresponding to various scattering paths.

For the edge-diffraction component, the waveform varies depending on the incident and diffracted directions. In the frequency domain, this variation of the waveform is interpreted as the frequency dependence of the phase of the diffracted waves. Here it is synthesized in the time domain from a table of possible combinations of these angles. Only two waveforms computed using the FDTD method are used as the reference waveforms for the diffracted waves. These are: the full reflection from a plane (which is just an inverted waveform of the incidence wave) and the scattering from the top of a thin ridge. Then the edge-diffraction waveforms for various combinations of the incident and diffracted waves are generated by using the FDTD method and are fitted by a linear combination of the two reference waveforms. The best-fit amplitude weights of the reference waveforms are stored in a matrix of the incident and diffracted angles and are used to synthesize the estimated diffraction waves.

The advantage of this method is not only that it provides a fast way of generating the desired diffraction waveform, but also that

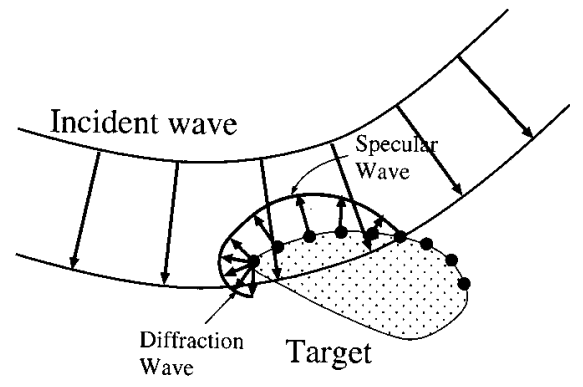

Fig. 2. Scattering of rays in the extended ray-tracing method from a finite object. Rays denoted by arrows constitute a ray group, and a diffraction wave is generated when the ray group intersects an edge of the target.

the table of the weights of the two reference waveforms is independent of the actual incident waveform. A change of the incident waveform due to dispersion or frequency-dependent reflection is realized by applying a filter function, as explained later. The same filter then must be applied to the two reference waveforms, but the weights of the waveforms remain unchanged for any desired diffraction wave.

As described in the previous section, the shape of objects is expressed as a smooth curve connecting discrete points that are located at an interval of about $\lambda / 10$. If the object has a sharp corner in the midst of its surface, additional diffracted waves should be generated at that point in order to represent the scattered wave properly. Although in principle it is possible to introduce such components, the current program simply neglects its effect. As a result, the reflected rays are directed to two widely separated directions around the corner, and the scattered field intensity is estimated as zero. The lower limit of the radius of curvature, for which the current algorithm works properly, is found to be about $\lambda / 10$ and has little dependence on the angle of apex for the region of less than $150^{\circ}$. It was also found that reducing the interval between the variable points that describe the object shape does not help in improving this lower limit, since the waveform of the diffraction component is substantially different from that of the reflection component.

\section{IMAGING IN A NondisPersive MEDIUM}

We first examine the characteristics of the algorithm for the case of the conductive target embedded in a uniform lossless nondispersive medium.

We assume a case in which the radar sensor scans linearly along the ground surface and transmits a monocycle pulse at a fixed interval. We neglect the effect of the ground, since it can be removed from the data fairly easily by subtracting the DC component in the direction of the scan or by applying the median filter to the data of the scan direction [8]. Fig. 3 shows the process of reconfiguring the shape of a perfectly conducting cylinder. Squares indicate the location of antennas, each of which is used for both transmission and reception and alternately in a monostatic operation. The abscissa and the ordinate is expressed in the unit of the radar wavelength at the center frequency of the transmitted pulse. The shaded circle represents the cross section of the given cylinder, and the small circles along its border indicate the reconstructed image. The simulated data is generated by using the FDTD method with the 

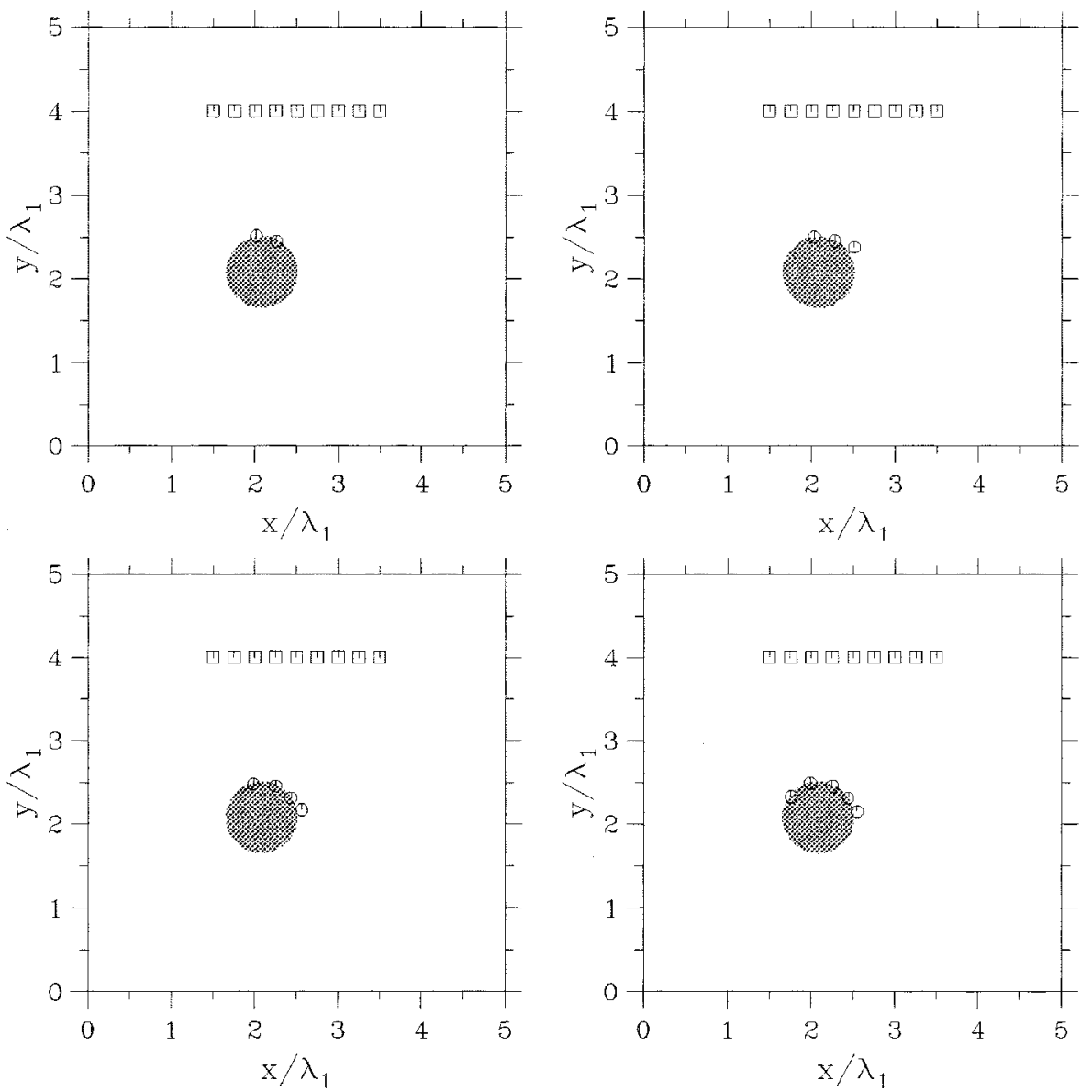

Fig. 3. Evolution of the model shape in the estimation process of a conducting cylinder.

second-order absorbing boundary [12] and is compared with the estimated received signal computed from the reconstructed image using the extended ray-tracing algorithm. Fig. 4 shows the residual of the fitting in each iteration. Similar results are obtained for other simple shapes, such as a conducting plate.

\section{TEST-SITE EXPERIMENTS}

We also made test-site experiments, which confirmed the performance of the proposed algorithm examined by this simulation. Fig. 5 shows the configuration of the test site. The test site is a wooden box of $90 \mathrm{~cm} \times 90 \mathrm{~cm} \times 180 \mathrm{~cm}$ filled with dry sand of the dielectric constant $\epsilon_{\mathrm{r}}=1.8$, which is determined by measuring the delay of echoes from thin aluminum pipes embedded at two known depths.

An aluminum pipe of $16.5 \mathrm{~cm}$ in diameter is embedded perpendicular to the long axis of the test site at a depth of $23.5 \mathrm{~cm}$. The surface of the sand is covered with a plate of acrylic resin of $5 \mathrm{~mm}$ thickness so that the scanning of the radar sensor can be made smoothly. The radar used is a commercial model IRS-150 developed by Komatsu Co. for detecting iron reinforcement in the concrete wall. It generates a monocycle pulse of 1-ns width, and oversamples the echo at 0.023 -ns intervals up to $6 \mathrm{~ns}$ by staggering the sampling gate. The $10-\mathrm{dB}$ bandwidth of the transmitted pulse covers $0.5-2.0 \mathrm{GHz}$.

The range profiles are averaged and recorded at every $5 \mathrm{~mm}$ of horizontal scan, which is detected by the rotation of the wheel on
Transition of the Residual

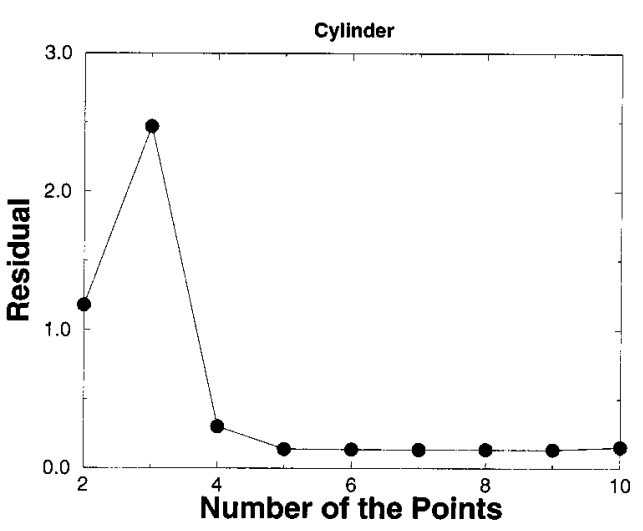

Fig. 4. Transition of the residual of fitting in each iteration of the imaging as the number of variable points is increased.

the sensor unit. Two bowtie antennas of 20-cm length are stored side-by-side in a shielded cavity filled with absorbing material and are used for transmission and reception, respectively.

Fig. 6 shows the result of the imaging. The abscissa and the ordinate are expressed in the unit of the wavelength $(22 \mathrm{~cm})$ of the nominal center frequency of $1 \mathrm{GHz}$ in the medium. Although the data are recorded over the entire scan length of $180 \mathrm{~cm}$, only the portion shown by squares in the figure (at which significant echo from the pipe was identified) was used for the imaging. 


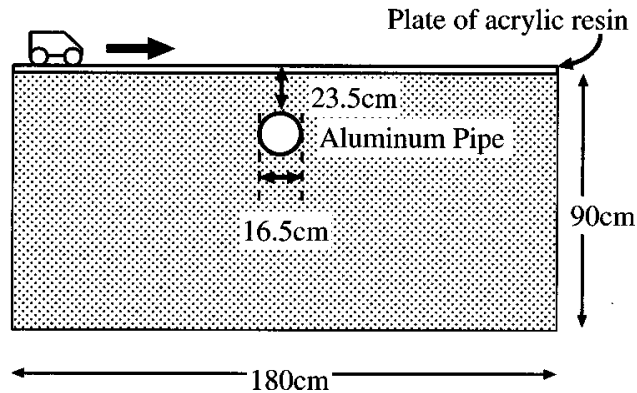

Fig. 5. Configuration of the test-site experiment.

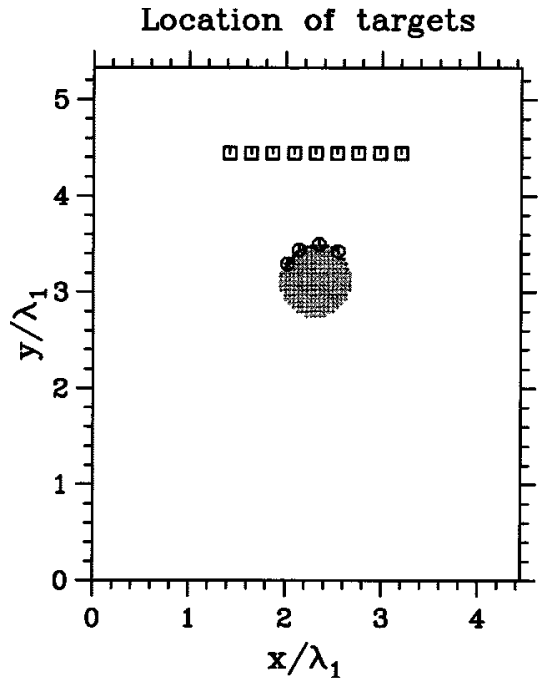

Fig. 6. Imaging of the cross section of an aluminum cylinder embedded in the test site.

In order to measure the overall impulse response of the system including the transmit and receive antennas and the characteristics of the medium, the echo waveform from a thin aluminum pipe, whose cross section can be safely regarded as a point compared to the radar wavelength, was first measured and used as the reference waveform for the echo from a point target. The extension of the target image was terminated on both sides of the apex of the pipe when it exceeds $1 / 4$ wavelength over the reflection boundary along the surface of the target. It is clearly shown that the upper surface of the pipe is precisely reconstructed.

Fig. 7 shows the result of an alternate experiment using an aluminum plate of $200-\mathrm{mm}$ width. The plate was embedded at various inclination angles. It was found that the shape was accurately reconstructed as far as the specular-reflection echo was received by the antenna. When the inclination angle becomes exceedingly large, the reconstructed image approaches that of a point target.

\section{IMAGING IN A DISPERSIVE MEDIUM}

Next, we examine the performance of the proposed algorithm for the case of a conductive object embedded in a uniform but lossy and dispersive medium. The attenuation and dispersion of the transmitted waveform is taken care of by applying proper filter functions, which are synthesized in the frequency domain. The model parameters to be determined are the permittivity and

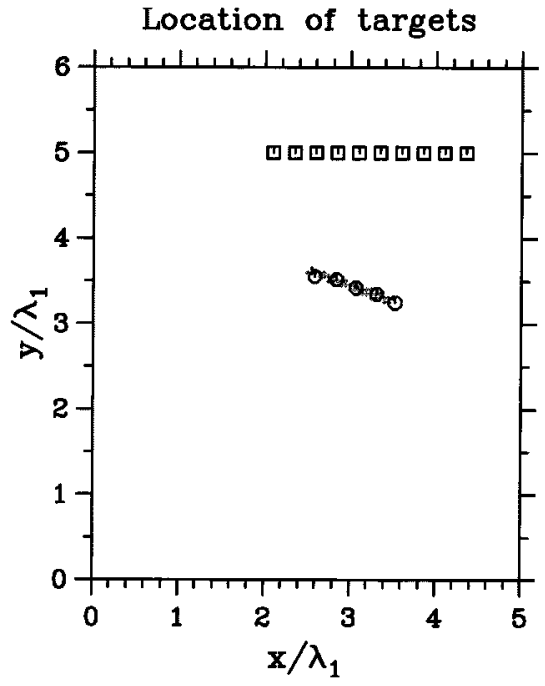

Fig. 7. Imaging of a slanted aluminum plate at the test site.

conductivity of the medium, their frequency derivatives, and the location of several points that characterize the outer contour of the object. Among the medium parameters, only the mean permittivity is assumed to be known, as was the case in the previous section. Other parameters are set to zero in the initial guess and are adjusted in the course of the recursive fitting.

The performance of the developed algorithm is quantitatively examined by numerical simulations. The simulated data are generated using the frequency-dependent FDTD method [13]. In this case, the complex permittivity of the medium is expressed by

$$
\epsilon_{\mathrm{r}}=a+\frac{c}{1+j \omega b}
$$

where $a, b$, and $c$ are the constants representing the medium characteristics, and $\omega$ is the angular frequency. The phase velocity $v$, attenuation constant $\beta$, and conductivity $\sigma$ are approximately expressed in terms of these constants as

$$
\begin{aligned}
& v=c_{0} / \sqrt{a+\frac{c}{1+\omega^{2} b^{2}}} \\
& \beta=60 \pi \frac{\epsilon_{0} \omega^{2} b c}{1+\omega^{2} b^{2}} / \sqrt{a+\frac{c}{1+\omega^{2} b^{2}}} \\
& \sigma=\frac{\epsilon_{0} \omega^{2} b c}{1+\omega^{2} b^{2}}
\end{aligned}
$$

where $c_{0}$ is the speed of the radio wave, and $\epsilon_{0}$ is the permittivity of the free space.

In the extended ray tracing, the received waveform is generated when a ray group passes a receiver position. Then the attenuation is computed as a function of frequency according to the estimated medium parameters, their frequency derivatives, and the distance the ray group traveled in the medium. The filter function is generated from these frequency characteristics, converted to the time domain, and applied to the reference waveform.

Target shapes assumed are conductive cylinders and plates whose size is a few wavelengths at the center frequency of the pulse, as was the case in previous sections. Fig. 8 compares 


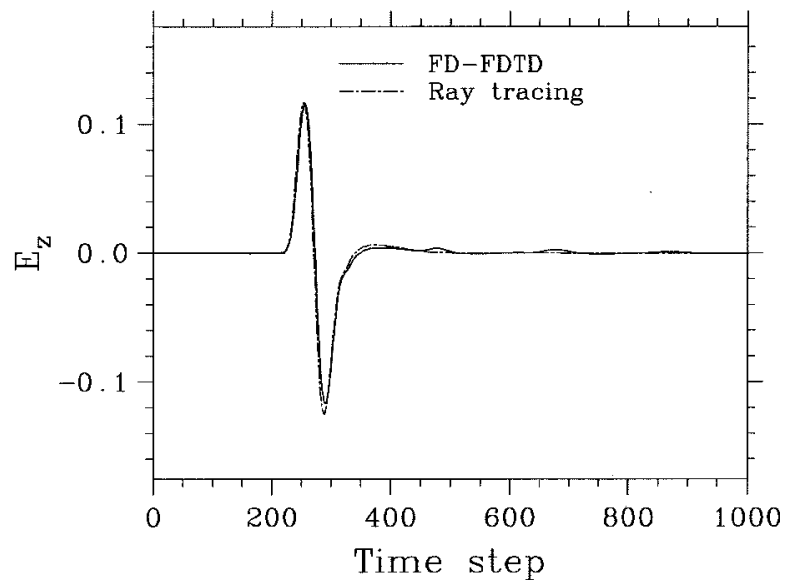

Fig. 8. Simulated received waveform by FD-FDTD method (solid line) and by the extended ray-tracing method (dot-dashed line) from a conductive cylinder embedded in a lossy-dispersive medium.

the scattered waveform of the FD-FDTD method and the developed ray-tracing method. The signal is computed for the case of a monocycle pulse scattered from a conductive cylinder embedded in a lossy-dispersive medium. The medium parameters used are for typical dry rock, namely

$$
\begin{aligned}
v & =1.36 \times 10^{8} \quad(\mathrm{~m} / \mathrm{s}) \\
\beta & =23.3 \quad(\mathrm{~dB} / \mathrm{m}) \\
\sigma & =3.16 \times 10^{-2} \quad(\mathrm{~S} / \mathrm{m}) .
\end{aligned}
$$

The phase velocity is roughly constant in the frequency band of $0.5-2 \mathrm{GHz}$, while the attenuation has a gradient of 23 $\mathrm{dB} / \mathrm{m} / \mathrm{GHz}$. The developed ray-tracing algorithm generates the estimated scattered wave roughly 100 times faster than the FD-FDTD method.

Fig. 9 shows the result of reconstruction of the same conducting cylinder as treated in Fig. 3 but embedded in the lossydispersive medium. This figure clearly shows that the developed model-fitting algorithm works almost equally well as for the case of nondispersive medium, and the shape of the upper surface of the targets is precisely reconstructed. The program was run on a UNIX workstation (SUN SPARCstation 20 with a single HyperSPARC $200 \mathrm{MHz}$ CPU), and it took $580 \mathrm{~s}$ of CPU time. Since the fastest workstation currently available in the market is already several times faster than this machine, the analysis of this level of complexity will become considered as a quasirealtime job in a very near future.

Current simulations assume that the electric field is perpendicular to the plane of interest for the transmitted and received waves, so the creeping wave which may contain the information of the lower side of the target is very weak compared with the direct scattering from the upper surface.

Here we treated the case in which the dispersion is mostly that of the attenuation and the phase velocity is roughly constant over the considered frequency range. If the medium has a strong dispersion in the phase velocity, it will become harder to synthesize the desired waveform from the reference waveforms. Fortunately, however, most types of rock have almost constant phase velocity in $200 \mathrm{MHz}-1 \mathrm{GHz}$ frequency range [14], so the present assumption can be made safely.

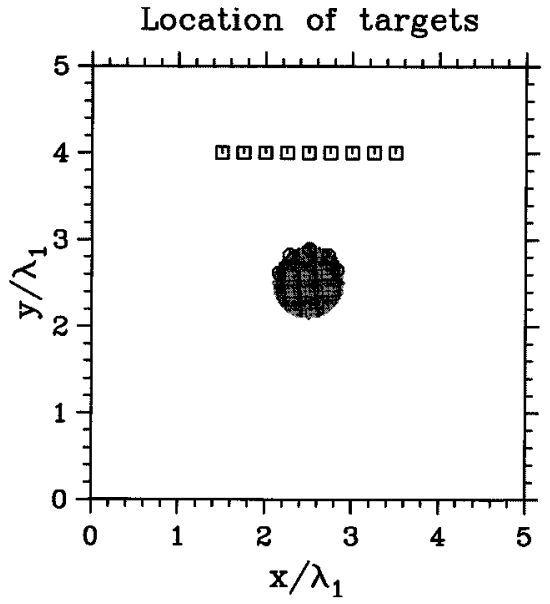

Fig. 9. Estimation of a cylinder in a lossy-dispersive medium.

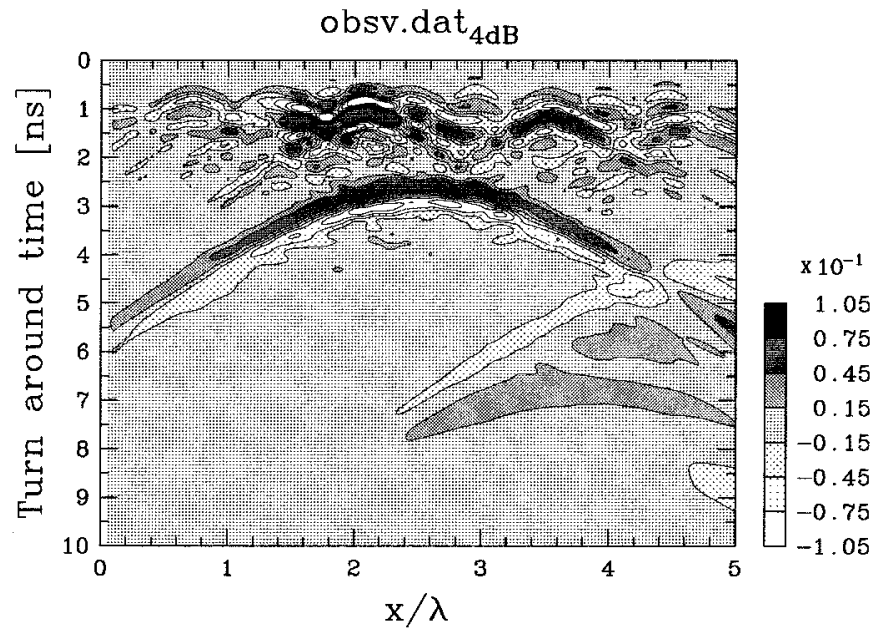

Fig. 10. Simulated echo from a cylinder in a lossy-dispersive medium with strong clutters $(S / C=4 \mathrm{~dB})$.

\section{EFFECT OF CLUTTERS}

In order to examine the tolerance of the algorithm against clutters, 200 point targets with various permittivity are randomly embedded in the simulated medium. The background medium is the same lossy-dispersive medium as assumed in the previous section.

Fig. 10 shows the simulated echoes from a cylinder of $0.8 \lambda$ in diameter in a strong clutter environment. The hyperbola peaked at $x=2.5 \lambda$, the delay of $2.5 \mathrm{~ns}$ is the reflection from the cylinder, and other echoes are from the point scatterers representing the clutter. The signal-to-clutter (S/C) ratio is controlled by adjusting the standard deviation of the distribution of the permittivity of random points and is set to $4 \mathrm{~dB}$ for this case.

Fig. 11 shows the simulated waveform at a point of $x=1.5 \lambda$. The desired echo from the cylinder shows up from about 250 time steps, but the clutters at shallower locations appear stronger than the desired echo. The clutter echoes at deeper locations are not only suppressed by the attenuation, but also low-pass filtered due to the dispersion of the medium.

The same model-fitting algorithm was applied to the cases with various magnitude of the clutter. No special means were taken to remove clutter echoes in the first and the second step 


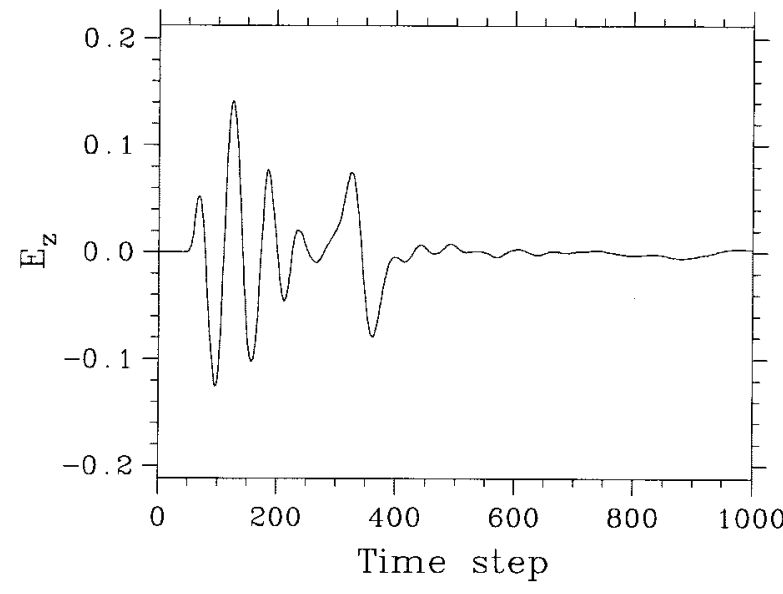

Fig. 11. Signal waveform at $x=1.5 \lambda$ in Fig. 10 .

of the algorithm. It was found that the algorithm can accurately reconstruct the target shape for an S/C ratio of larger than about $10 \mathrm{~dB}$. The reason that fairly strong clutters do not affect the estimate is because the nonlinear least squares fitting finds the local minimum around the initial guess, which is affected only by clutters that occurs at the same time window as the desired echo.

The shape estimation gradually deteriorates when the clutter level is further increased, and the points that represent the outer contour of the target start to deviate from the true locations. However, the size of the target can be still correctly estimated with S/C of up to about $4 \mathrm{~dB}$. Fig. 12 shows the estimated shape of the cylinder for the $\mathrm{S} / \mathrm{C}$ of $4 \mathrm{~dB}$. The shaded circle indicates the given shape of the target, and small circles shows the estimated outer contour. Compared to Fig. 9 for the same medium without clutter, The right-most point of the estimated shape does not agree with the given contour, but the horizontal extent of the points well represents the target size. This overall agreement keeps unchanged for different random numbers for the clutter distribution as far as the $\mathrm{S} / \mathrm{C}$ ratio is the same. It confirms the robustness of the proposed algorithm against disturbances.

When the clutter level exceeds this threshold, the algorithm mistakes the strongest clutter point for the target. For a higher clutter level, it is necessary to take care of strong individual clutters. We have separately developed an algorithm that considers the target to be a group of point targets [7]. Although it is in principle possible to combine this method with the current algorithms, the number of parameters may become too large to make a stable estimate.

\section{SUMMARY}

A 2-D shape-estimation algorithm was developed for the situation of a solid conductive target embedded in a lossy-dispersive medium. An extended ray-tracing method is developed for fast estimation of the scattering from an object approximately the size of the radar wavelength. Its accuracy and its range of applicability has been examined.

The performance of the algorithm has been tested first for nondispersive cases with numerical simulations and test-site experiments. Numerical simulations for the lossy and dispersive medium confirmed the capability of the algorithm, which

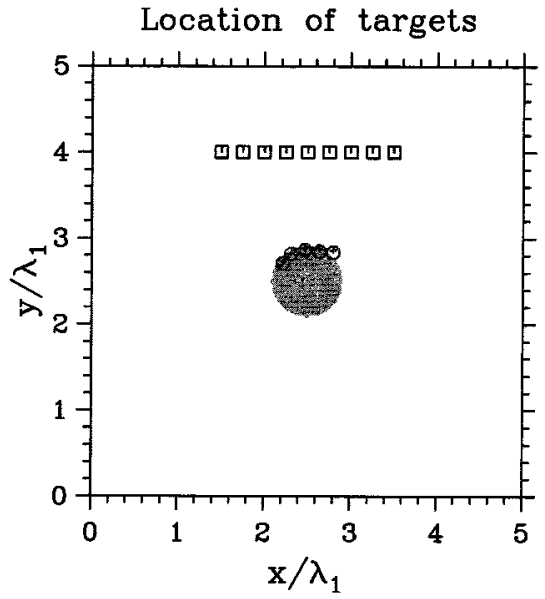

Fig. 12. Same as Fig. 9, but in a strong clutter environment $(S / C=4 d B)$.

showed equally good performance in the case of the nondispersive medium. The simulation with clutters also revealed the robustness of the algorithm even under a fairly strong clutter environment.

The major limitation of the algorithm is that we have to model the given situation precisely. The current model assumes a uniform medium, which is still too simple to deal with realistic cases of field excavations. We are currently extending the algorithm to fit the medium consisting of multiple layers with arbitrary boundaries.

\section{REFERENCES}

[1] D. L. Mensa, High Resolution Radar Cross-Section Imaging. Norwood, MA: Artech House, 1991.

[2] C. E. Baum, E. J. Rothwell, K.-M. Chen, and D. P. Nyquist, "The singularity expansion method and its application to target identification," Proc. Inst. Elect Eng. F, vol. 79, pp. 1481-1492, Oct. 1991.

[3] P. Chaturvedi and R. G. Plumb, "Electromagnetic imaging of underground targets using constrained optimization," IEEE Trans. Geosci. Remote Sensing, vol. 33, pp. 551-561, May 1995.

[4] C. Torres-Verdìn and T. M. Habashy, "Rapid 2.5-dimensional forward modeling and inversion via a new nonlinear scattering approximation," Radio Sci., vol. 29, no. 4, pp. 1051-1979, 1994.

[5] J. V. Candy and C. Pichot, "Active microwave imaging: A model-based approach," IEEE Trans. Antennas Propagat., vol. 39, pp. 285-290, Mar. 1991.

[6] IEICE Trans. Commun., vol. E76-B, pp. 1546-1551, Dec. 1993.

[7] T. Wakayama, T. Sato, and I. Kimura, "High-resolution radar image reconstruction using an arbitrary array," IEICE Trans. Commun., vol. E76-B, pp. 1305-1312, Oct. 1993.

[8] T. Sato, K. Takeda, T. Nagamatsu, T. Wakayama, I. Kimura, and T. Shinbo, "Automatic signal processing of front monitor radar for tunneling machines," IEEE Trans. Geosci. Remote Sensing, vol. 35, pp. 354-359, Mar. 1997.

[9] D. W. Marquardt, "An algorithm for least squares estimation of nonlinear parameters," SIAM J. Appl. Math., pp. 431-441, Nov. 1963.

[10] T. Wakayama, T. Sato, and I. Kimura, "Radar image reconstruction by discrete model fitting in a layered inhomogeneous medium," in Proc. 5th Int. Conf. Ground Penetrating Radar, 1994, pp. 223-237.

[11] E. F. Knott, J. F. Shaeffer, and M. T. Tuley, Radar Cross Section, 2nd ed. Norwood, MA: Artech, 1993.

[12] G. Mur, "Absorbing boundary conditions for the finite-difference approximation of the time-domain electromagnetic-field equations," IEEE Trans. Electromag. Compat., vol. EMC-23, pp. 377-384, Apr. 1981.

[13] R. Luebbers, K. S. Kunz, R. B. Standler, and M. Schneider "A frequency-dependent finite-difference time-domain formulation for dispersive materials," IEEE Trans. Electromag. Compat., vol. EMC-32, pp. 222-227, Mar. 1990

[14] G. Turner, "Propagation deconvolution," in Proc. 4th Int. Conf. Ground Probing Radar, 1992, pp. 85-93. 
Toru Sato (M'92), photograph and biography not available at the time of publication.

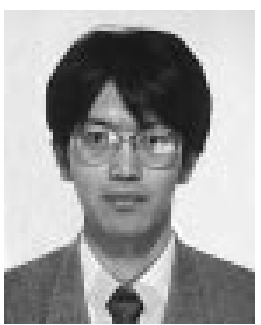

Toshio Wakayama received the B.E., M.E., and $\mathrm{Ph} . \mathrm{D}$ degrees in electrical engineering from Kyoto University, Japan, in 1990, 1992, and 1995, respectively.

He joined Mitsubishi Electric Corporation, Tsukaguchi, Amagasaki, Japan, in 1995. His research interest is radar system design and radar signal processing.

Dr. Wakayama is a member of the Institute of Electronics, Information, and Communication Engineers.

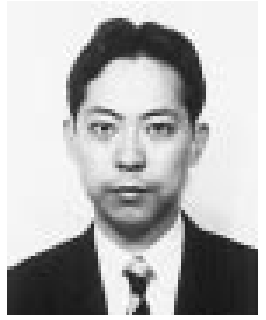

Kazuhisa Takemura received the B.E. and M.E. degrees in electrical engineering from Kyoto University, Japan, in 1995 and 1997, respectively.

He joined Kansai Electric Power Co. Ltd., Osaka, Japan, in 1997. His research interests are in subsurface radar signal processing. 